Pesq. Vet. Bras. 35(3):297-303, março 2015 DOI: $10.1590 / \mathrm{S} 0100-736 \mathrm{X} 2015000300014$

\title{
Análise quantitativa da ecogenicidade testicular pela técnica do histograma de ovinos da baixada ocidental maranhense ${ }^{1}$
}

\author{
Elzivânia G. Silva ${ }^{2 *}$, Mysa T.C. Gonçalves ${ }^{3}$, Sâmara C.C. Pinto ${ }^{4}$, Diego M. Soares ${ }^{4}$, \\ Rudson A. Oliveira ${ }^{5}$, Flávio R. Alves ${ }^{6}$, Adriana V.C. Araújo ${ }^{5}$ e Porfírio C. Guerra ${ }^{5}$
}

\begin{abstract}
Silva E.G., Gonçalves M.T.C., Pinto S.C.C., Soares D.M., Oliveira R.A., Alves F.R., Araújo A.V.C. \& Guerra P.C. 2015. [Quantitative analysis of testicular echogenicity by technical histogram in sheep of the western lowland region of Maranhão, Brazil.] Análise quantitativa da ecogenicidade testicular pela técnica do histograma de ovinos da baixada ocidental maranhense. Pesquisa Veterinária Brasileira 35(3):297-303. Departamento das Clínicas Veterinárias, Universidade Estadual do Maranhão, Cidade Universitária Paulo VI, Cx. Postal 9, São Luís, MA 65630-410, Brazil. E-mail: elzivania_us@hotmail.com

Ultrasound is a diagnostic imaging method that allows a no invasive evaluation of different organs and body structures. However, the personal evaluation of images is one of the major hampers in using this technique. In order to reduce this drawback, it is necessary to find out tools that can help diminish the divergence in the interpretation of sonographic findings. Therefore, this study aimed to identify features of the parenchyma's and mediastinum's testis of sheep echogenicity using the technique of gray scale histogram. 30 animals were divided into three groups according to age (FE): three to six months (FE1), seven to 12 months (FE2), 13 to 18 months (FE3). The testicular scans were performed in the frontal, sagital and transverse views, and the gray scale histograms were analyzed. It was observed that such parenchymal as the mediastinum testis echogenicity gradually increased with the aging of the animals, with mean and standard deviation of $95.00 \pm 19.05$ and $94.35 \pm 18.82$ for the echogenicity parenchyma of the right and left antimere, respectively, and $127.95 \pm 12.97$ to $126.59 \pm 11.78$, for the right and the left mediastinum. The technique of gray scale histogram was an efficient method for determination of testicular echogenicity, enabling the establishment of normal standards that may assist future research in monitoring testicular development as well as for detecting pathologies. For exclusive regimes of extensive farming, as the western lowland region of Maranhão, is a valuable tool for use in social projects of the State to attend the family farm.
\end{abstract}

INDEX TERMS: Testicular echogenicity, mediastinum testis, testicular parenchyma, microlithiasis, testicular tunics.

${ }^{1}$ Recebido em 11 de setembro de 2014.

Aceito para publicação em 8 de março de 2015.

${ }^{2}$ Programa de Pós-Graduação em Ciência Animal, Universidade Federal do Piauí (UFPI), Campus Ministro Petrônio Portella, Ininga, Teresina, PI 64049550, Brasil. *Autor para correspondência: elzivania_us@hotmail.com

3 Departamento de Química e Biologia, Universidade Estadual do Maranhão (UEMA), Rua Godofredo Viana s/n, Centro, Imperatriz, MA 65901480, Brasil. E-mail: mysa_taty@yahoo.com.br

${ }^{4}$ Programa de Pós-Graduação em Ciência Animal, UEMA, Cidade Universitária Paulo VI, Cx. Postal 9, São Luís, MA 65630-410, Brasil. E-mail: samara.cristinecosta@yahoo.com

${ }^{5}$ Departamento das Clínicas Veterinárias, UEMA, Cidade Universitária Paulo VI, Cx. Postal 9, São Luís, MA 65630-410. E-mail: porfirio_uema@ yahoo.com.br

${ }^{6}$ Departamento de Morfofisiologia, UFPI, Campus Universitário Ministro Petrônio Portella, Ininga, Teresina, PI 64049-550. E-mail: flavioribeiro@ ufpi.edu.br
RESUMO.- A ultrassonografia é um método de diagnóstico por imagem que permite a avaliação de diferentes órgãos e estruturas corpóreas de maneira não invasiva. No entanto, a avaliação subjetiva das imagens caracteriza um dos grandes entraves na utilização desta técnica de diagnóstico, havendo necessidade de mecanismos que minimizem a subjetividade do exame e a divergência na interpretação dos achados ultrassonográficos. Desta forma este trabalho objetivou caracterizar a ecogenicidade do parênquima e mediastino testicular de ovinos utilizando a técnica do histograma escala-cinza. Foram utilizados 30 animais divididos em três grupos de acordo com a faixa etária (FE): de três a seis meses (FE1), sete a 12 meses (FE2), 13 a 18 meses (FE3) e realizadas varreduras testiculares nos planos frontal, sagital e transversal, elaborando ao final um histo- 
grama a partir das imagens ultrassonográficas. Observou-se que tanto a ecogenicidade do parênquima quanto a do mediastino testicular aumentaram gradativamente com a progressão das idades dos animais, com média e desvio-padrão de 95,00 $\pm 19,05$ e 94,35 $\pm 18,82$ para a ecogenicidade do parênquima do antímero direito e esquerdo, respectivamente, e $127,95 \pm 12,97$ para o mediastino direito e $126,59 \pm 11,78$ para o esquerdo. A técnica do histograma escala-cinza demonstrou ser um método eficiente na determinação da ecogenicidade testicular, possibilitando o estabelecimento de padrões de normalidade que venham a auxiliar pesquisas futuras no monitoramento do desenvolvimento testicular bem como na detecção de patologias. Para a regimes exclusivos de criação extensiva, como na baixada maranhense, representa ferramenta valiosa para sua utilização em projetos sociais do Estado que atendem a agricultura familiar.

TERMOS DE INDEXAÇÃO: Ecogenicidade testicular, mediastino testicular, parênquima testicular, microlitíase, túnicas testiculares.

\section{INTRODUÇÃO}

0 primeiro registro da utilização da ultrassonografia na medicina veterinária ocorreu no ano 1966, quando Ivan Lindahl diagnosticou prenhez em ovelhas (Lindahl 1996). É uma técnica de diagnóstico que permite, num curto espaço de tempo, avaliar diversos fenômenos da fisiologia reprodutiva de forma alternada ou em observação contínua de um evento dinâmico, contribuindo, para obtenção de importantes informações morfológicas (Cruz \& Freitas 2001).

Vários fatores podem influenciar no estabelecimento do diagnóstico ultrassonográfico, como a experiência do avaliador, aparelho utilizado, frequência do transdutor, regulagem de profundidade, ganho e contraste (Kenneth et al. 1986, Zweibel 1995). De acordo com Filho et al. (2009), a avaliação subjetiva das imagens constitui uma desvantagem do método, sendo que a impressão visual muitas vezes é insuficiente para determinar a ecogenicidade exata dos tecidos.

Para tentar evitar possíveis erros de interpretação e minimizar a subjetividade do exame, utilizam-se técnicas que quantificam a ecogenicidade e ecotextura como análise por sinal de vídeo (Waller et al. 2003), estimação da atenuação (Kenneth et al. 1986, Lu et al. 1997) e backscatter (Garra et al. 1984, Parker et al. 1984, Lu et al. 1997) e histograma dos níveis de cinza (Osawa \& Mori 1996, Maeda, Utsu \& Kihaile 1998, Lee et al. 2006).

Pela técnica do histograma, cada pixel da imagem sonográfica vai armazenar um sinal digital, determinado pela intensidade do eco refletido pelo tecido que está sendo avaliado. 0 programa elabora a representação gráfica do número de pixels, associado a cada nível de cinza presente na imagem (Queiroz 2006). A ecogenicidade é determinada pela quantificação dos ecos que retornam ao transdutor, enquanto que, a ecotextura, é determinada pela quantidade de pontos luminosos que mais se repetem na região selecionada e pela diferença da amplitude de ecos ultrassonográficos que retornam ao transdutor e formam a imagem selecionada (Maeda et al. 1998, Lee et al. 2006). Essa análise pode dar informações úteis sobre a estrutura e a função do testículo (Chandolia et al. 1997).

O histograma escala-cinza encontra-se disponível em alguns aparelhos ultrassonográficos comerciais, porém sua aplicação tem se restringindo ao meio acadêmico. Dessa forma, existe uma necessidade de padronização de valores, para utilização na prática clínica e em protocolos experimentais na Medicina Humana e Veterinária (Maeda et al. 1998).

Como os ovinos são considerados animais precoces no que diz respeito aos aspectos reprodutivos, esse estudo teve por objetivo avaliar ultrassonograficamente a ecogenicidade do parênquima e mediastino testicular por meio da análise quantitativa do histograma escala-cinza, fornecendo valores de normalidade para padronização da técnica nesses animais. Uma vez que os ovinos criados da baixada maranhense são criados extensivamente e sem recursos técnicos, este recurso pode complementar informações de valor a agricultura familiar por meio de uma captura rápida e em tempo real do padrão ultrassonográfico testicular nesses animais.

\section{MATERIAL E MÉTODOS}

0 estudo foi realizado, na cidade de São Bento $\left(02^{\circ} 40^{\prime} 00^{\prime \prime}\right.$ latitude Sul; 4443'17" longitude Oeste), localizada na mesorregião norte do Maranhão e microrregião ocidental da Baixada Maranhense, com altitude média de $2 \mathrm{~m}$ acima do nível do mar.

Foram avaliados 30 ovinos machos, sem padrão racial definido, com idades de três a dezoito meses, dividido em três grupos, de acordo com as faixas etárias: faixa etária 1 (FE1): de três a seis meses; faixa etária 2 (FE2): de sete a 12 meses; faixa etária 3 (FE3): de 13 a 18 meses. Os animais foram mantidos em sistema semi-intensivo, tendo acesso à pastagem nativa (Paspalum sp.) e água ad libidum. 0 estudo foi aprovado pelo Comitê de Ética do Curso de Medicina Veterinária, Universidade Estadual do Maranhão (UEMA), com protocolo de número 020/2011.

A avaliação ultrassonográfica dos testículos dos ovinos foi realizada mensalmente utilizando aparelho de ultrassom portátil (Kaixim ${ }^{\circledR} 5500$ ), com transdutor linear de 7,5 MHz de frequência, sendo os animais mantidos em estação e contidos manualmente, sem utilização de tranquilizantes, e os testículos examinados sem nenhuma tração.

Para avaliação da ecogenicidade do parênquima testicular (PT) e mediastino testicular (MT), foi aplicada uma camada de gel de carboximetilcelulose sobre a bolsa escrotal e o transdutor foi posicionado longitudinalmente ao maior eixo de cada testículo e realizadas varreduras eletrônicas nos planos sagital e frontal e avaliações transversais dos testículos direito e esquerdo. Para minimizar as variáveis que pudessem interferir nos resultados, todas as varreduras foram realizadas pelo mesmo profissional e o aparelho de ultrassom calibrado da mesma forma quanto ao posicionamento do foco principal e a intensidade de brilho e contraste.

As imagens capturadas foram transferidas para um dispositivo móvel de armazenamento, sendo posteriormente analisadas em computador por meio do histograma escala-cinza pelo software GIMP 2.8 (2012). Para elaboração do histograma selecionou-se três áreas de interesse em cada plano de varredura dos antímeros testiculares de cada animal, sendo ao final calculada a média das três aferições. A delimitação das áreas foi realizada utilizando um quadrado de $6,3 \mathrm{~mm}^{2}$ para o parênquima e $2 \mathrm{~mm}^{2}$ para o medias- 
tino testicular (Cardilli et al. 2009), com precaução para não selecionar imagem da pele, túnicas testiculares e epidídimo.

A escala utilizada para mensurar o percentual de tonalidade de cinzas (intensidade de pixels da imagem) variou de 0 a 255 , indicando respectivamente, imagem totalmente preta (parênquima testicular menos ecogênico) e imagem totalmente branca (parênquima testicular mais ecogênico).

Para avaliação estatística realizou-se a análise de variância (ANOVA) com teste t pareado para comparação das médias entre os antímeros testiculares, e teste de Tukey-Kramer para comparação entre as faixas etárias (FE1, FE2 e FE3). As análises foram realizadas utilizando o pacote estatístico GraphPad Instat. (2000) versão 3.05, com nível de significância mínima de 5\% (p>0,05).

\section{RESULTADOS}

O padrão ultrassonográfico do parênquima testicular dos ovinos sem padrão racial definido mostrou-se homogêneo em $80 \%$ dos animais independente do plano de varredura (Fig.1), não havendo diferença estatística significativa $(p>0,05)$ entre a ecogenicidade do parênquima do antímero direito $(95,00 \pm 19,05)$ e do esquerdo $(94,35 \pm 18,82)$. Também não foi verificada diferença estatística significativa $(p>0,05)$ quando comparada as médias das ecogenicidades do mediastino testicular direito $(127,95 \pm 12,97)$ e esquerdo $(126,59 \pm 11,78)$, apresentando quantidade de pixels próximos entre si.

O MT foi observado em $100 \%$ dos animais como uma linha hiperecóica central tanto nos planos sagital como frontal (Fig.1), com ecogenicidade maior que a do PT, apresentando variações na intensidade de pixels de acordo com a faixa etária dos carneiros avaliados. As túnicas testiculares (TT) foram visualizadas ultrassonograficamente como uma linha hiperecogênica brilhante, circundando o parênquima testicular e separando-o da bolsa escrotal (Fig.2).

Quando comparada a ecogenicidade do parênquima testicular pelo histograma escala-cinza em relação às faixas etárias, observou-se variação de 69,91 a 113,42 pixels,

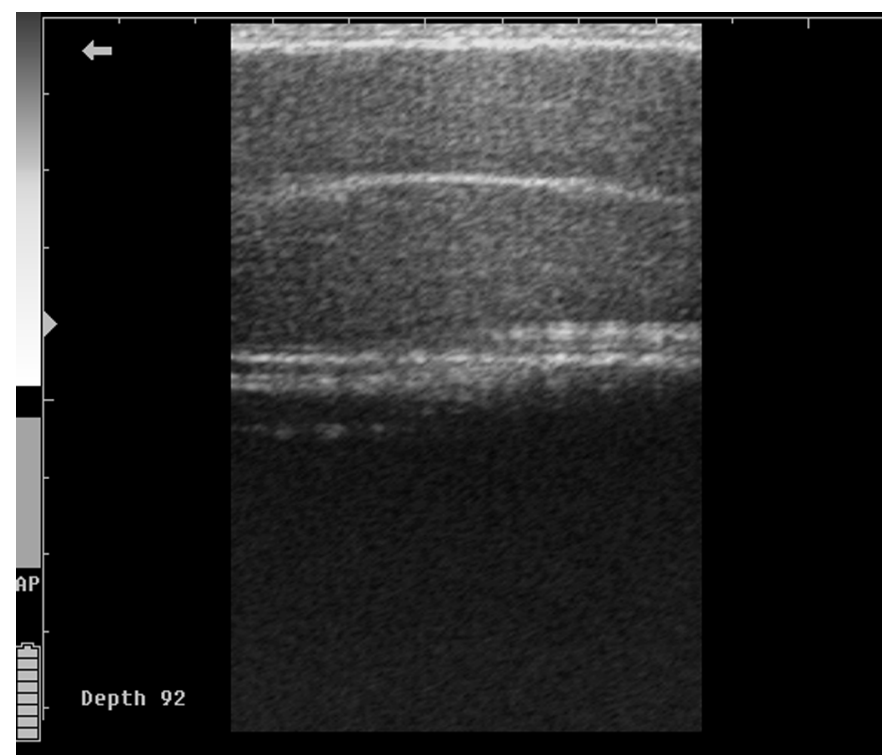

Fig.1. Imagem ultrassonográfica em plano sagital do parênquima testicular (PT) e mediastino testicular (MT). São Luís/MA, 2013.

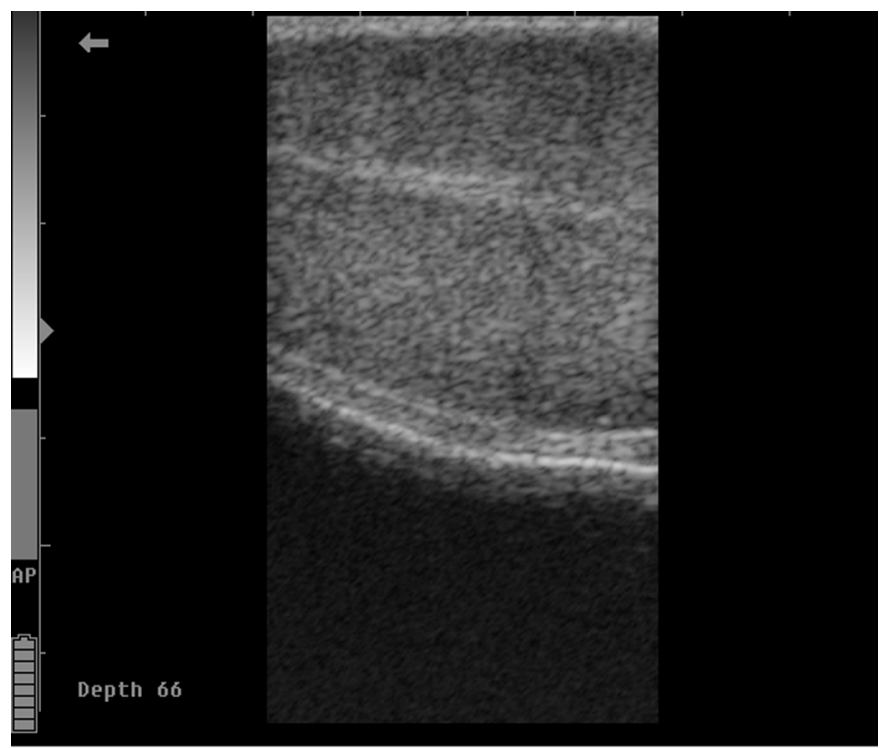

Fig.2. Imagem ultrassonográfica em plano sagital das túnicas testiculares (TT) e bolsa escrotal (BE). São Luís/MA, 2013.

com ecotextura homogênea em $80 \%$ dos animais (Quadro 1). Houve correlação positiva $(p<0,0001)$ da ecogenicidade em função da idade dos animais $(r=0,8433)$, com imagens de menor ecogenicidade nas primeiras avaliações, quando os animais apresentavam três meses, aumentando gradativamente até a última avaliação, quando aos 18 meses de idade, com a média dos três grupos diferentes $(\mathrm{p}<0,05)$ entre si.

Em relação ao mediastino testicular, identificado em todos os animais, notou-se evento semelhante ao observado no PT, mostrando-se mais ecogênico nos animais mais velhos, com variação de 100,16 a 143,35 pixels entre as idades de três a 18 meses, numa escala de zero a 255, com diferença estatística significativa $(\mathrm{p}<0,05)$ entre a média dos três grupos (Quadro 1).

Foram visualizados ultrassonograficamente em seis animais do estudo (20\%) pontos hiperecogênicos variando de 0,2 a $1,7 \mathrm{~mm}$ distribuídos no parênquima testicular de forma difusa (Fig.3) sem formação de sombreamento acústico, sendo imagem compatível com quadro de microlitíase testicular. Em todos os ovinos acometidos pela alteração foi possível contabilizar mais de cinco pontos hiperecóicos em ambos os antímeros testiculares da primeira à última avaliação ultrassonográfica.

\section{DISCUSSÃO}

A média da ecogenicidade dos testículos direito e esquerdo dos ovinos do presente estudo foi semelhante, apresentando quantidade de pixels próximos entre si. Estes achados corroboram aos trabalhos de Lavoipierre (2000), quando descreveram o padrão ultrassonográfico do testículo normal como homogêneo, onde ambos os antímeros devem apresentar mesma ecogenicidade, caso contrário, deve-se suspeitar de patologia.

Teixeira et al. (2011) avaliando ovinos das raças Dopper e Santa Inês utilizando a técnica do histograma demonstraram que não houve diferença estatística significativa entre 
Quadro 1. Valores médios e respectivos desvios-padrão das variáveis
ultrassonográficas testiculares de ovinos, sem padrão racial definido,
segundo a faixa etária, oriundos do município de São Bento/MA. São Luís, 2013

\begin{tabular}{|c|c|c|c|c|c|}
\hline \multirow[t]{2}{*}{ Variáveis testiculares } & & \multicolumn{3}{|c|}{ Faixa etária (meses) } & \multirow[t]{2}{*}{$\mathrm{P}$} \\
\hline & & FE1 & FE2 & FE3 & \\
\hline Ecogenicidade & TD & $69,91^{\mathrm{a}} \pm 10,25$ & $91,79^{b} \pm 14,56$ & $113,42^{c} \pm 9,83$ & $<0,0001$ \\
\hline Parênquima (pixels) ${ }^{1}$ & $\mathrm{TE}$ & $70,49^{a} \pm 11,25$ & $91,09^{b} \pm 15,46$ & $112,26^{c} \pm 7,51$ & $<0,0001$ \\
\hline Ecogenicidade & $\mathrm{TD}$ & $100,16^{\mathrm{a}} \pm 8,72$ & $127,49^{b} \pm 12,36$ & $143,35^{c} \pm 8,85$ & $<0,0001$ \\
\hline mediastino (pixels) ${ }^{1}$ & $\mathrm{TE}$ & $101,78^{a} \pm 9,53$ & $126,05^{\mathrm{b}} \pm 13,46$ & $142,68^{c} \pm 9,38$ & $<0,0001$ \\
\hline
\end{tabular}

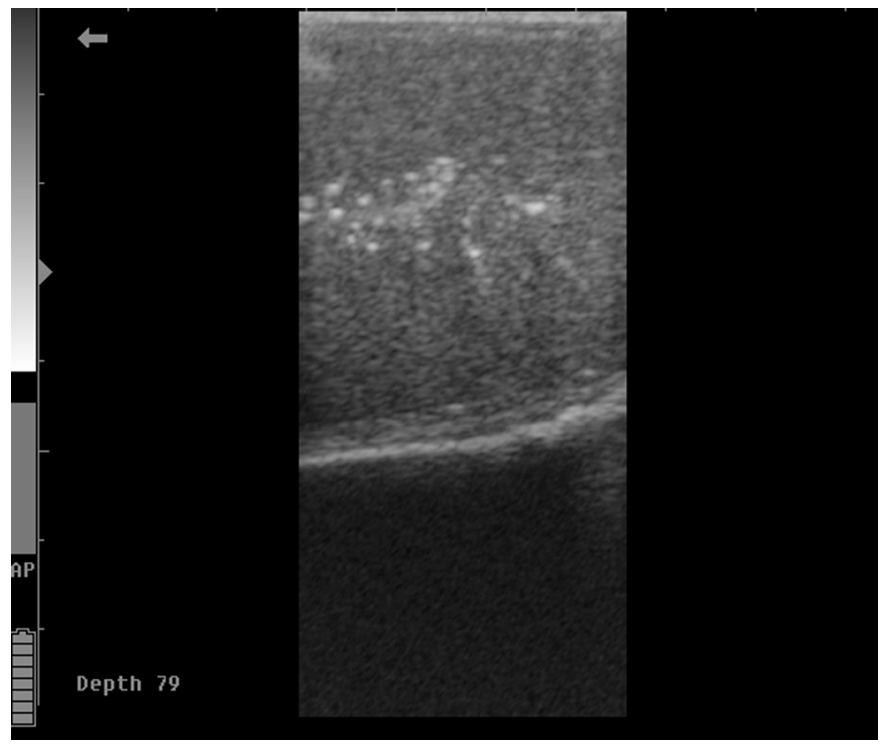

Fig.3. Imagem ultrassonográfica em plano frontal representando múltiplos pontos ecogênicos distribuídos difusamente no PT de um ovino sem padrão racial definido aos treze meses de idade, indicando quadro de microlitíase testicular. São Luís/ MA, 2013.

a ecogenicidade do parênquima dos testículos direito e esquerdo entre as espécies. Ao avaliarem testículos de bovinos jovens da raça Nelore criados em sistema intensivo, Cardilli et al. (2012) também obtiveram os mesmos achados, não encontrando diferença estatística significativa entre os antímeros direito e esquerdo.

Estudos realizados por Jucá et al. (2009) evidenciaram que o padrão ultrassonográfico dos testículos de carneiros da raça Santa Inês em diferentes idades, classificando-os segundo sua ecogenicidade em hipoecóicos de baixa e alta intensidade, sendo mais homogêneas ecogenicamente no testículo direito. 0 mediastino testicular foi descrito como difuso, moderadamente e altamente ecogênico, identificando uma maior predominância do padrão moderadamente ecogênico.

Em seus estudos, Andrade Moura et al. (2008) observaram uma variação de imagens hipoecóicas de baixa e alta intensidade no parênquima testicular nos grupos de ovinos da raça Santa Inês, havendo predominância das imagens hipoecóicas de baixa intensidade. Outros autores realizando avaliação da ecogenicidade do parênquima testicular de bovinos (Pechman \& Eilts 1987, Abdel-Razek \& Ali 2005,
Cardilli et al. 2009), caprinos (Ahmad, Noakes \& Subandrio 1991), cães (Green 1996), suínos (Clark et al. 2003) e ovinos (Gouletsou et al. 2003), classificaram o parênquima testicular como homogêneo, de ecogenicidade moderada ou hipoecóica.

Observa-se que a nomenclatura ultrassonográfica utilizada para determinação da ecogenicidade tanto do parênquima quanto do mediastino testicular é variada entre autores, necessitando, portanto, de uma normatização com o intuito de estabelecer padrões que possam facilitar a avaliação testicular dos ovinos de diferentes raças e em diferentes regiões.

0 mediastino testicular foi observado em $100 \%$ dos animais como uma linha hiperecóica central tanto nos planos sagital como frontal, com ecogenicidade maior que a do PT, apresentando variações na intensidade de pixels de acordo com a faixa etária dos carneiros avaliados. Gouletsou et al. (2003) conceituaram o MT como uma massa de tecido fibroso contendo numerosos túbulos finos na parte central do órgão. Jucá et al. (2009) definiram como uma estrutura central linear hiperecóica de ecogenicidade variável, observado ultrassonograficamente nos planos sagital e transverso, descrição esta que está em concordância com o observado neste estudo.

Gouletsou et al. (2003) afirmam que a presença e a forma do mediastino testicular são determinantes na impressão ecogênica do parênquima. Em seu trabalho, o mediastino testicular foi observado somente em $87 \%$ dos animais examinados, e a ecogenicidade do mediastino aumentou progressivamente com a idade dos animais.

As túnicas testiculares foram visualizadas por ultrassonografia como uma linha hiperecogênica brilhante, circundando o parênquima testicular e separando-o do escroto, dados que somam aos observado por Cardilli et al. (2010) em suas avaliações de bovinos da raça Nelore, que descreveram as túnicas testiculares como uma linha hiperecóica que circunda todo o parênquima testicular em plano transversal. Não houve possibilidade de identificação das túnicas separadamente, uma vez que não existia presença de líquido entre elas (Pechman \& Eilts 1987). Gouletsou et al. (2003) afirma que a camada de fluido normal entre as túnicas nunca excede dois milímetros de espessura, e que, quando excedem esse valor, podem ser consideradas anormais.

Foi observado aumento da ecogenicidade do parênquima testicular com a progressão da idade dos animais, 
corroborando com os achados de Gouletsou et al. (2003) e Andrade Moura et al. (2008), que da mesma forma que Chandolia et al. (1997) explicam que esse evento ocorre em virtude do conjunto de transformações no interior dos testículos com a chegada da puberdade. No primeiro momento é observada proliferação celular, iniciando-se então a divisão das espermatogônias e a formação das demais células maduras da espermatogênese. Posteriormente, acontece aumento da densidade das células, caracterizada pela formação das espermátides e consequente transformação em espermatozoides. Segundo Ahmad et al. (1991) o exame ultrassonográfico é uma ferramenta útil no monitoramento de mudanças progressivas que ocorrem nos testículos.

Andrade et al. (2012) avaliando ultrassonograficamente testículos de carneiros mestiços, verificaram PT de baixa ecogenicidade até os 140 dias de idade, com aumento identificado a partir de 168 dias. Os mesmos autores também encontraram uma fase em que a ecogenicidade testicular diminuiu, atribuindo este fato a formação do lúmen dos túbulos seminíferos, término da formação das células de Sertoli, e consequente, produção de fluido por estas células, necessários a espermatogênese. Tapping e Cast (2008) verificaram diferenças na ecogenicidade do PT entre animais púberes e pré-puberes, havendo predominância de imagens de baixa ecogenicidade nos cordeiros pré-púberes e moderada nos púberes.

Chandolia et al. (1997) examinando ultrassonograficamente testículos de 10 bovinos da raça Hereford a cada duas semanas nos planos transversal e longitudinal, observaram aumento da ecogenicidade do parênquima testicular com o avanço das semanas, não havendo diferença estatística entre os planos. 0 número de pixels aumentou do nascimento até oito semanas de idade; de oito a 20 semanas foi verificado um platô; um segundo aumento foi observado de 20 a 30 semanas, seguido por um pequeno declínio após 30 semanas. Uma última elevação na escala de pixels foi encontrada a partir da quadragésima semana.

Avaliando a região do mediastino testicular pelo histograma de acordo com as faixas etárias, observou-se aumento na média as aferições de acordo com o avanço da idade dos animais. Segundo Dyce et al. (1990) e Hamm \& Fobbe (1995) esse evento seria explicado devido a ocorrência de mudanças anatômicas importantes e consideráveis nos túbulos seminíferos que formam no mediastino a rede do testículo (rete testis), e que, com o aumento da idade do animal, se tornam mais longos e "retorcidos", aumentam em diâmetro e formam um lúmen.

Não foi possível a avaliação ultrassonográfica da cabeça e do corpo do epidídimo em decorrência do tipo de transdutor utilizado (linear), porém a visualização da cauda do epidídimo ocorreu em $100 \%$ dos animais estudados, apresentando-se como uma imagem de menor ecogenicidade quando comparada ao parênquima testicular, corroborando com os achados de Pechman \& Eilts (1987), Ahmad et al. (1991), Gouletsou et al. (2003) e Amorim (2010) que descreveram a cauda do epidídimo como uma estrutura com ecogenicidade reduzida em relação ao parênquima testicular.
Amorim (2010) também descreveu não ter realizado a avaliação ultrassonográfica da cabeça e do corpo do epidídimo em decorrência da dificuldade de contato entre o transdutor linear e as estruturas. Andrade Moura et al. (2008), avaliando testículos e glândulas sexuais acessórias de ovinos, também utilizando transdutor do tipo linear, relataram que a avaliação do epidídimo foi preterida pelo fato da dificuldade de adaptação deste sobre o órgão, inviabilizando o foco e a geração da imagem.

Em 20\% dos animais avaliados foram identificados presença de múltiplos pontos hiperecogênicos distribuídos em região do PT em ambos os antímeros testiculares, caracterizando a presença de microlitíase testicular. Essa patologia é caracterizada pela presença de calcificações intratubulares compostas por múltiplas camadas de fibras colágenas, sem a formação de sombra acústica. A doença está frequentemente associada a neoplasias testiculares, e, quando diagnosticada, requer acompanhamento ultrassonográfico anual (Dogra et al. 2003, Sakamoto et al. 2006). Embora essas formações não apresentem sombra acústica posterior, ocasionalmente podem gerar artefatos em cauda de cometa ou um padrão cintilante ao doppler colorido (Roy \& Tuchmann 2003).

Foi possível identificar presença de quantidade superior a cinco pontos hiperecóicos em cada testículo dos animais avaliados, até o último momento das avaliações. Segundo Janzen et al. (1992) para o diagnóstico ultrassonográfico, o critério mais aceito para confirmação de microlitíase testicular é a identificação de um número mínimo de cinco microcalcificações por campo de imagem. A presença de mais de cinco pontos de calcificação é considerada anormal, sendo compatível com o desenvolvimento de alterações neoplásicas em humanos (Sakamoto et al. 2006).

A etiologia e os significados clínicos da microlitíase testicular ainda são desconhecidos, todavia a doença está associada com múltiplas enfermidades como: criptorquidismo, transtornos da diferenciação sexual, alterações vasculares (torção testicular e calcificação vascular), subfertilidade, enfermidades inflamatórias e neoplasias testiculares (Villalobos et al. 2008). Nos ovinos do presente estudo não foram verificadas quaisquer tipos de alterações clínicas que permitissem fazer esta associação.

Gomes et al. (2011) avaliando dois bodes da raça Saanen de quatro anos de idades, que estavam sendo utilizados em estudos sobre vascularização testicular, encontraram pontos hiperecogênicos com tamanho variando de 0,5 a 1,5mm distribuídos de forma difusa no parênquima testicular, sendo contabilizados mais de cinco pontos hiperecóicos em cada testículo dos animais. Esses autores relataram ainda dificuldade em observar a atividade da artéria testicular próximo aos pontos de calcificação.

0 exame ultrassonográfico testicular tem por objetivo avaliar a estrutura anatômica interna e determinar a ecogenicidade do parênquima e mediastino testicular (Pechman \& Eilts 1987, Cartee et al. 1989, Chandolia et al. 1997, Feliciano Silva et al. 1997, Kastelic 1997, Gàbor et al. 1998, Clark et al. 2003), demonstrando elevada importância no diagnóstico complementar de patologias testiculares, como neoplasias, calcificações, inflamações e degenera- 
ções (Ahmad et al. 1991, Geraghty et al. 1998, Ganem et al. 1999, Howlett et al. 2000, Derogee et al. 2001, Miller \& Sidhu 2002, Sartori et al. 2002).

A subjetividade do exame ultrassonográfico de rotina é um fator limitante para uma precisa avaliação por este método de exame complementar (Osawa \& Mori 1996, Lu et al. 1997, Maeda et al. 1998, Nicoll 1998, Lee et al. 2006). Ultrapassar esta barreira é o principal objetivo da técnica quantitativa do histograma dos níveis de cinza (Vescovi et al. 2009).

\section{CONCLUSÕES}

A técnica do histograma escala-cinza demonstrou ser um método eficiente na determinação da ecogenicidade testicular durante as mudanças progressivas que ocorrem no interior dessas gônadas nas diferentes fases reprodutivas dos ovinos e, aliado ao exame ultrassonográfico, permite identificar alterações patológicas sem manifestações clínicas evidentes.

Estes dados podem oferecem clínicas importantes aos criadores que adotam modelos de criação extensiva na baixada maranhense, constituindo-se como uma ferramenta valiosa para sua utilização em projetos sociais do Estado que atendem a agricultura familiar.

Agradecimentos.- À Fundação de Amparo a Pesquisa e Desenvolvimento Científico do Maranhão (FAPEMA) pelo apoio financeiro.

\section{REFERÊNCIAS}

Abdel-Razek A. \& Ali A. 2005. Development changes of bull (Bos taurus) genitalia as evaluated by caliper and ultrasonography. Reprod. Domest. Anim. 40:23-27.

Ahmad N., Noakes D.E. \& Subandrio A.L. 1991. B-mode real time ultrasonographic imaging of the testis and epididymis of sheep and goats. Vet. Rec. 12:491-496.

Amorim A.K.G.A. 2010. Ultrassonografia dos testículos e epidídimos de carneiros jovens na fase Peri-puberal. Dissertação de Mestrado em Ciência Veterinária, Universidade Federal Rural de Pernambuco, Recife. 90p.

Andrade Moura J.C., Jucá A.F., Gusmão A.L., Bittencourt T., Pinho T.G. \& Barbosa C.M.P. 2008. Ecotextura testicular do carneiro Santa Inês. Hora Vet. 27:19-22.

Andrade A.K.G., Soares A.T., Cartaxo F.Q., Peña-Alfaro C.E. \& Guerra M.M.P. 2012. Achados ultrassonográficos nos testículos e epidídimos de carneiros deslanados jovens e clinicamente sadios. Arq. Bras. Med. Vet. Zootec. 64:371-379.

Cardilli D.J., Toniollo G.H., Pastore A.A., Canola J.C. \& Mercadante M.E.Z. 2009. Alterações do padrão ultrassonográfico do parênquima testicular em bovinos jovens da raça Nelore. Acta Scient. Vet. 37:367-370.

Cardilli D.J., Toniollo G.H., Pastore A.A., Canola J.C., Mercadante M.E.Z. \& Oliveira J.A. 2010. Padrão ultrassonográfico do parênquima, mediastino e túnicas testiculares em bovinos jovens da raça Nelore. Ciênc. Anim. Bras. 11:899-905.

Cardilli D.J., Toniollo G.H., Pastore A.A., Canola J.C., Oliveira J.A. \& Mercadante M.E.Z. 2012. Ultrassonografia testicular em bovinos jovens da raça Nelore criados em sistema extensivo. Arq. Bras. Med. Vet. Zootec. 64:75-82.

Cartee R.E., Gray B.W., Powe T.A. \& Whitesides J. 1989. Preliminary implications of B-mode ultrasonography of the testicles of beef bulls with normal breeding soundness examinations. Theriogenology 31:1149-1157.

Chandolia R.K., Bartlewski P.M., Beard A.P., Rawlings N.C. \& Pierson R.A. 1997. Ultrasonography of the developing reproductive tract in RAM lambs effects of a GnRH agonist. Theriogenology 48:99-117.
Clark S.G., Schaeffer D.J. \& Althouse G.C. 2003. B-mode ultrasonographic evaluation of paired testicular diameter of mature boars in relation to average total of sperm numbers. Theriogenology 60:1011-1023.

Cruz J.F. \& Freitas V.J.F. 2001. A ultra-sonografia em tempo real na reprodução de caprinos. Ciênc. Anim. 11:45-53.

Derogee M., Bevers R.F.M., Prins H.J., Jonges T.G.N., Elbers F.H. \& Boon T.A. 2001. Testicular microlithiasis, a premalignant condition: prevalence, histopathologic findings, and relation to testicular tumor. Urology 57:1133-1137.

Dogra V.S., Gottlieb R.H., Oka M. \& Rubens D.J. 2003. Sonography of the scrotum. Radiology 237:18-36.

Dyce K.M., Sack W.O. \& Wensing C.J.G. 1990. 0 aparelho urogenital, p.133165. In: Ibid. (Eds), Tratado de Anatomia Veterinária. Guanabara Koogan, Rio de Janeiro.

Feliciano Silva A.E.D., Kastelic J.P., Unanian M.M., Freitas A.R. \& Cook R.B. 1997. Ultra-sonografia de machos Nelore na fase peripuberal. Revta Bras. Reprod. Anim. 21:34-36.

Filho O.O.S., Nardozza L.M.M., Junior E.A., Rolo L.C., Camano L. \& Moron A.F. 2009. Estudo da cicatriz uterina de cesariana avaliada pelo histograma escala-cinza. Revta Assoc. Méd. Bras. 56:99-102.

Gàbor G., Sasser R.G., Kastelic J.P., Ménez M., Falkay G., Bozó S., Volgyicsik J., Bárany I., Hidas A., Szász Jr F. \& Boros G. 1998. Computer analysis of video and ultrasonographic images for evaluation of bull testes. Theriogenology 50:223-228.

Ganem J.P., Workamn K.R. \& Shaban S.F. 1999. Testicular microlithiasis associated with testicular pathology. Urology 53:209-213.

Garra B.S., Shawkwe T.H., Nassi M. \& Russell M.A. 1984. Ultrasound attenuation measurements of liver in vivo using a commercial sector scanner. Ultrason Imag. 6:396-407.

Geraghty M.J., Lee F.T.J.R., Bernsten S.A., Gilchrist K., Pozniak M.A. \& Yandow D.J. 1998. Sonography of testicular tumors and tumor-like conditions: a radiologic-pathologic correlation. Crit. Rev. Diagn. Imaging 39:1-63.

Gomes W.A., Silva I.C.C., Pelinca M.A., Camargo N.I., Silva E.C.B., Silva S.V., Batista A.M., Guerra M.M.P. \& Costa F.S. 2011. Calcificação testicular em caprinos Saanen: relato de dois casos. Med. Vet. 5:13-16.

Gouletsou P.G., Amiridis G.S., Cripps P.J., Deligiannis K., Saratsis P. \& Fthenakis G.C. 2003. Ultrasonographic appearance of clinically healthy testicles and epididymes of rams. Theriogenology 59:1959-1972.

GraphPad Instat. 2000. Versão 3.05, 32 bit fow Win 95/NT. GraphPad Software, San Diego, California, EUA.

Green R.W. 1996. Kidneys, p.197-210. In: Ibid. (Ed.), Small Animal Ultrasound. Lippincott Williams and Wilkins, Philadelphia.

Hamm B. \& Fobbe F. 1995. Maturation of the testis: ultrasound evaluation. Ultrasound Med. Biol. 21:143-147.

Howlett D.C., Marckbank N.D.P. \& Sallomi D.F. 2000. Ultrasound of the testis. Clin. Radiol. 55:595-601.

Janzen D.L., Mathieson J.R., Marsh J.I., Cooperberg P.L., Del Rio P., Golding R.H. \& Rifkin M.D. 1992. Testicular microlithiasis: sonographic and clinical features. Am. J. Roentgenol.158:1057-1060.

Jucá A.F., Moura J.C.A., Gusmão A.L., Bittencourt T.C., Nascimento M.C. \& Barbosa C.M. 2009. Avaliação ultrassonográfica dos testículos e das glândulas sexuais anexas de carneiros Santa Inês. Ciênc. Anim. Bras. 10:650-659.

Kastelic J.P. 1997. Relationships between pixel intensity of testicular ultrasonograms and sperm morphology in Nelore and Canchim bulls. Revta Bras. Reprod. Anim. 21:40-42.

Kenneth J.W., Taylor M.D., Riely C.A., Hammers L., Flax S., Weltin G., Garcia-Tsao G., Conn H.O., Kuc R. \& Barwick K.W. 1986. Quantitative US attenuation in normal liver and in patients with diffuse liver disease: importance of fat. Radiology 160:65-71.

Lavoipierre A.M. 2000. Ultrasound of the prostate and testicles. World J. Surg. 24:198-207.

Lee H.C., Choi J.W., Kim K.A., Seo T.S., Lee J.M. \& Park C.M. 2006 Usefulness 
of standard deviation on the histogram of ultrasound as a quantitative value for hepatic parenchymal echo texture; preliminary study. Ultrasound Med. Biol. 32:1817-1826.

Lindahl I.L. 1966. Detection of pregnancy in sheep by means of ultrasound, Nature. 212:642-643.

Lu Z.F., Zaqzebski J.A., O’Brien R.T. \& Steinberg H. 1997. Ultrasound attenuation and backscatter in the liver during prednisone administration. Ultrasound Med. Biol. 23:1-8.

Maeda K., Utsu M. \& Kihaile P.E. 1998. Quantification of sonographic echogenicity with grey-level histogram width: A clinical tissue characterization. Ultrasound Med. Biol. 24:225-234.

Miller F.N.A.C. \& Sidhu P.S. 2002. Does testicular microlithiasis matter? A review. Clin. Radiol. 57:883-890.

Nicoll R.G., O’Brien R.T. \& Jackson M.W. 1998. Qualitative ultrasonography of the liver in obese cats. Vet. Radiol. Ultrasound 39:47-50.

Osawa H. \& Mori Y. 1996. Sonographic diagnosis of fatty liver using a histogram technique that compares liver and renal cortical echo amplitudes. J. Clin. Ultrasound 24:25-29.

Parker K.J., Lerner R.M. \& Waag R.C. 1984. Attenuation of ultrasound: magnitude and frequency dependence for tissue characterization. Radiology 153:785-788.

Pechman R.D. \& Eilts B.E. 1987. B-mode ultrasonography of the bull testicle. Theriogenology 27:431-441.

Queiroz J.E.R. 2006. Introdução ao processamento digital de imagens. Rev. Inf. Teor. Apl. 13:11-42.
Roy C. \& Tuchmann C. 2003. Échografie scrotale-patho-logies kystiques, tumorales et non tumorales rares (part 2). J. Radiol. 84:667-680.

Sakamoto H., Saito K., Shichizyo T., Ishikawa K., Igarashi A. \& Yoshida H. 2006. Color Dopler ultrasonography as a routine clinical examination in male fertility. Int. J. Urology 13:1073-1079.

Sartori R., Prestes N.C., Canavessi A.M.O., Kempinas W.G. \& Rosa G.J.M. 2002. Avaliações ultra-sonográfica, macroscópica e histológica da biopsia testicular em ovinos. Arq. Bras. Med. Vet. Zootec. 54:1-12.

Tapping C.R. \& Cast J E. 2008. Scrotal ultrasound: a pictorial review. Ultrasound 16:226-233.

Teixeira P.P.M., Oliveira M.E.F., D'amato C.C., Rodrigues L.F.S. \& Vicente W.R.R. 2011. Avaliação ultrassonografia da ecogenicidade do parênquima testicular como exame complementar no andrológico de carneiros. Nucl. Anim. 3:31-36.

Vescovi L.A., Monteiro J.N.M., Santos W.G., Oliveira D.C., Borlini D.C., Machado F.M., Filho S.M. \& Costa F.S. 2009. Ultrassonografia quantitativa do baço de gatos normais. Veterinária in Foco 7:4-10.

Villalobos G.M., Negrete P.O., Mendez P.C., Fuentes C.R., Sotomayor Z.M. \& Feria B.G. 2008. Importancia de microlitiasis en ultrasonido de neoplasias de células germinales de testículos. Actas Urol. 32:190-193.

Waller K.R., Knipp B.S., O’Brien R.T. \& Zaqzebski J.A. 2003. The effect of body wall on video signal analysis measurements. Vet. Radiol. Ultrasound 44:222-225.

Zweibel W.J. 1995. Sonographic diagnosis of diffuse liver disease. Seminars in Ultrasound, CT and MRI, 16:8-15. 